\title{
“Eu não sou um anjo azul": a sexualidade na perspectiva de adolescentes autistas
}

\author{
"I am not a blue angel": \\ Sexuality from the perspective of autistic adolescentes
}

\begin{abstract}
Aline Veras Morais Brilhante (https://orcid.org/0000-0002-3925-4898) ${ }^{1}$
Leila Maria de Andrade Filgueira (https://orcid.org/0000-0002-2210-4498) ${ }^{1}$

Samuel Verter Marinho Uchôa Lopes (https://orcid.org/0000-0002-1231-7051) ${ }^{1}$

Nathalie Barreto Saraiva Vilar (https://orcid.org/0000-0003-0512-630X) ${ }^{1}$

Lívia Rocha Mesquita Nóbrega (https://orcid.org/0000-0002-5077-5723) ${ }^{1}$

Ana Juarina Magalhães Verissimo Pouchain (https://orcid.org/0000-0002-4713-4626) ${ }^{1}$

Luiz Carlos Gabriele Sucupira (https://orcid.org/0000-0003-1680-601X) ${ }^{1}$
\end{abstract}

${ }^{1}$ Programa de Pós-

Graduação em Saúde

Coletiva, Universidade de

Fortaleza. Av. Washington

Soares 1321/S-1, Edson

Queiroz. 60811-905

Fortaleza CE Brasil.

alineveras@unifor.br

\begin{abstract}
Despite the difficulties in living their sexualities, the sexual education of autistic people is often neglected. In this sense, this research aims to identify autistic people's demands on their sexualities, in line with the neurodiversity paradigm. This qualitative research was carried out from September 2017 to October 2018, with 14 autistic children aged 15 to 17 enrolled in regular schools. Data were collected by semi-structured interviews and analyzed according to the thematic content analysis. Two analytical categories were identified: "discursive processes and the "blue angel' imagery"; and "diversity in diversity: the sexuality of autistic people as singular processes". The results show that, while autistic people grow physically and sexually according to the typical development stages, some singularities should not be ignored. However, the construction of false beliefs encourages the denial of autistic people's sexuality. We can conclude that the establishment of effective actions of sexual education and support to the sexuality of the autistic person requires a paradigmatic change anchored in the social model of disability.
\end{abstract}

Key words Sexual education, Sexuality, Autism
Resumo Apesar das dificuldades enfrentadas na vivência de suas sexualidades, a educação sexual de pessoas autistas costuma ser negligenciada. Neste sentido, esta pesquisa objetiva identificar as demandas de autistas sobre sua sexualidade, alinhando-se ao paradigma da neurodiversidade. Trata-se de uma pesquisa de natureza qualitativa realizada de setembro de 2017 a outubro de 2018, com 14 autistas oralizados, com idades de 15 a 17 anos, matriculados em escolas regulares. A coleta dos dados foi realizada por entrevistas semiestruturadas e avaliadas segundo a análise temática de conteúdo. Identificaram-se 2 categorias analíticas: "processos discursivos e a imagética do 'anjo azul" e "diversidade na diversidade: a sexualidade de autistas como processos singulares". Os resultados que embora pessoas autistas se desenvolvam fisicamente e sexualmente de acordo com os estágios típicos de desenvolvimento, existem singularidades que não deveriam ser ignoradas. Todavia, a construção de falsas crenças fomenta a negação da sexualidade de pessoas autistas. Conclui-se que o estabelecimento de ações efetivas de educação sexual e de suporte à sexualidade da pessoa autista requer uma mudança paradigmática, que ancorese no modelo social de deficiência.

Palavras-chave Educação sexual, Sexualidade, Autismo 


\section{Introdução}

Apesar do crescente interesse da literatura científica em relação ao autismo, este é ainda um Campo em disputa, permeado por divergências e controvérsias ${ }^{1}$. Partindo de diferentes paradigmas, alguns pesquisadores têm explorado a esfera psicossexual do autismo e ressaltado a necessidade de analisá-la não apenas em uma perspectiva externa (de pais, cuidadores ou especialistas), mas principalmente em âmbito pessoal ${ }^{2}$. Nesse contexto, é salutar a ampliação de pesquisas que analisem a sexualidade dessa população a partir das experiências subjetivas ${ }^{2,3}$. Torna-se particularmente interessante o aumento de produções científicas baseadas em um paradigma cunhado no seio da comunidade autista, como é o caso da Neurodiversidade ${ }^{3}$.

O deslocamento do paradigma psicanalítico clássico iniciado por Leo Kanner e o avanço das neurociências culminou na emergência de múltiplos modelos de interpretação do autismo e, consequentemente, em diversas abordagens terapêuticas. Conquanto sejam diferentes em suas bases teóricas, a maioria das abordagens ancora-se em terapias comportamentais e psicofarmacológicas, em busca da cura ou do controle das características autísticas, incluindo os chamados "comportamentos sexuais inapropriados"2,4.

Em oposição a essa perspectiva, emerge o movimento da Neurodiversidade. Este paradigma - ao contrário do que afirmam alguns críticos - reconhece o autismo como uma deficiência, embora também o considere uma condição neurodiversa ${ }^{3}$. Alinhada ao modelo social de deficiência, a Neurodiversidade tem como pressuposto chave que todas as formas de diversidade neurológica devem ser respeitadas ${ }^{3,5}$. Deste modo, as terapêuticas deveriam buscar não uma cura, mas centrar-se em maximizar habilidades, fornecer suporte socioemocional e fomentar estratégias diante das demandas individuais e ambientais ${ }^{6,7}$. Nesse contexto, uma das reinvindicações permanentes entre ativistas da Neurodiversidade referese ao direito da pessoa autista sobre seu corpo e sua sexualidade ${ }^{3,8}$, independente do nível de limitações, refutando a crença de que autistas seriam pessoas assexuadas ou "eternas crianças".

Independente do paradigma, contudo, a literatura é escassa em pesquisas sobre as necessidades das pessoas autistas no que se refere à esfera sexual ${ }^{8}$. Em grande medida, esse vácuo associa-se ao entendimento da deficiência como incapacidade $^{2,6}$ e ao silenciamento e à desqualificação das falas dos próprios autistas ${ }^{4,8,9}$. Neste sentido, este artigo objetiva analisar as demandas de pessoas autistas sobre sua sexualidade, alinhando-se ao paradigma da neurodiversidade.

\section{Metodologia}

Trata-se de uma pesquisa de natureza qualitativa realizada com pessoas autistas, no período entre setembro de 2017 e outubro de 2018, cujos dados preliminares foram apresentados no $8^{\circ}$ Congresso Ibero-Americano em Investigação Qualitativa - CIAIQ ${ }^{10}$. Os participantes foram recrutados por chamamento público em mídias sociais que congregam autistas e familiares. A opção por participar da pesquisa foi espontânea.

Os critérios de inclusão foram: ser autista oralizado ou utilizar técnica de comunicação alternativa que permitisse responder a entrevista. Foram excluídos adolescentes menores de 18 anos sem autorização dos responsáveis e autistas que não dispunham de ferramentas comunicativas que possibilitasse a entrevista. Atenderam ao convite 14 autistas oralizados, entre 15 e 17 anos, sendo oito do gênero masculino, cinco do feminino e um participante que se identificou como gênero neutro. Vale ressaltar que todos estavam matriculados em escolas regulares. Um dos participantes, apesar de oralizado, utilizava eventualmente tecnologias de comunicação aumentativa e alternativa por meio de aplicativo de celular, devido a episódios de mutismo seletivo. Essa tecnologia não foi necessária nesta pesquisa. Nove participantes residiam em Fortaleza, três em Maracanaú, um em Caucaia e um no Eusébio, todos municípios do estado do Ceará, Brasil.

A coleta dos dados foi realizada por entrevistas semiestruturadas que abordaram as dificuldades enfrentadas pelos autistas na vivência da sua sexualidade e suas demandas no que se refere à educação sexual. As entrevistas foram realizadas pela mesma pesquisadora que, além de ser mãe de uma criança autista, possui traços que a caracterizam como parte do Fenótipo Ampliado do Autismo (FAA) ${ }^{11}$. Essa particularidade contribuiu para o estabelecimento do rapport, sendo um facilitador no processo das entrevistas.

Os dados foram analisados segundo a análise temática de conteúdo ${ }^{12,13}$ partindo da neurodiversidade como referencial teórico. A fim de manter o anonimato, os informantes foram identificados por pseudônimos escolhidos por eles. $\mathrm{O}$ projeto desta pesquisa foi aprovado pelo Comitê de Ética e Pesquisa da Universidade de Fortaleza. 


\section{Resultados}

Identificaram-se 2 categorias analíticas: processos discursivos e a imagética do "anjo azul"; e diversidade na diversidade: a sexualidade de autistas como processos singulares.

\section{Processos discursivos e a imagética do "anjo azul"}

A dificuldade de pais e professores em reconhecer autistas como pessoas sexuadas foi recorrente nos discursos. Para os informantes, essa resistência é fomentada pela introjeção de falsas crenças sobre o autismo. A falácia de que autistas são eternas crianças é contestada por Elric (16 anos):

Eu não sou criança. Eu tenho uma namorada. Mas ainda é difícil para as pessoas aceitarem isso, porque elas querem que a gente seja criança pra sempre. É a tal lenda da idade mental.

Elric, cuja namorada também é autista, considera-se uma pessoa com autonomia limitada: relata restrições para sair de casa sem suporte, dificuldades de adaptação escolar e distúrbio do processamento sensorial, que comprometem as atividades de vida diária. Ao trazer essas questões, Elric declara:

Eu sei que eu tenho minhas limitações. Mas isso não dá a ninguém o direito de me impedir de ter uma namorada. E minha família tentou isso no começo. Ainda hoje eles tratam como se fosse uma brincadeira de criança.

Sakura (17 anos) ratifica a crítica de Elric ao conceito de idade mental e à infantilização de pessoas autistas, principalmente daquelas com comprometimento cognitivo:

eu fico pistola quando escuto alguém dizer que 'fulano tem a idade mental de uma criança de 5 anos.' O que está implícito ai é que essa pessoa precisa ser tutelada para sempre. [...] Eu tenho amigas com déficit cognitivo mais severo que o meu. Elas têm desejo do mesmo jeito, mas são limitadas porque a sociedade não permite que elas tenham direito ao próprio corpo.

Sakura afirma que nunca houve diálogo intrafamiliar sobre sexualidade e que sempre que tentou abordar o tema foi interrompida:

Eu tentei perguntar, tentei conversar. Mas todo mundo se apavora e só sabe dizer que eu não tenho malícia pra essas coisas porque eu sou inocente. Mas minha irmã mais nova que não é autista namora e ninguém diz nada. Eu odeio essa história que autista é inocente.

O estereótipo de inocência e a negação da sexualidade de pessoas autistas ganha uma ima- gética a partir de uma construção metafórica. A expressão "anjo azul", amplamente disseminada para caracterizar pessoas autistas, atua como processo discursivo, normatizando sua infantilização e contribuindo para a invisibilidade do autismo feminino, como ilustra a fala de Jasmin (15 anos):

Eu não sou um anjo azul. Não nasci pra anjo. Não sou assexual. E sou menina. Esse azul aí surgiu por que as pessoas acham que autismo é mais prevalente em meninos e não é. A gente, menina, demora muito mais pra conseguir o diagnóstico. $E$ essa história desse anjo azul dificulta ainda mais.

Na mesma perspectiva, Kalel (17 anos), complementa que o imaginário do "anjo azul" contribui para a negação da sexualidade das pessoas autistas:

Nós somos pessoas, a gente tem desejo, a gente sente as coisas, mas é difícil para nossos pais entenderem talvez até por que eles foram condicionados a nos olharem como anjos azuis.

\section{Diversidade na diversidade: a sexualidade de autistas como processos singulares}

As falas de nossos colaboradores evidenciam que as experiências e demandas das pessoas autistas quanto à sua sexualidade são tão diversas quanto o espectro.

Conquanto Kalel (17 anos) seja considerado um jovem com altas habilidades acadêmicas, o mesmo relata dificuldades de socialização:

No meu caso eu tenho muita dificuldade em lidar com as pessoas. Eu sou muito rígido com minhas rotinas e as pessoas tendem a não gostar de mim e acham que eu não me interesso por elas. Sou tido como antipático.

Kalel tem um irmão dois anos mais jovem, também autista com graves limitações de autonomia e diversas condições associadas. Sobre o irmão, que recebeu o diagnóstico de "autismo clássico", Kalel nos fala:

$\hat{E}$ diferente comigo e com ele [...]. As pessoas querem que ele seja criança. Mas ele tem 15 anos, já descobriu o prazer genital. E aí vão fazer o quê? Castrar o menino? Olha que tem gente que faz isso. Castração química. É terrível isso.

Seguindo a linha narrativa, Kalel reforça que o direito à sexualidade de pessoas autistas deveria ser respeitado independentemente do nível de autonomia. Contudo, o que ele observa em seu cotidiano é de um lado a infantilização do irmão, de outro, a invisibilidade de suas demandas:

No caso do meu irmão, as pessoas tendem a achar que ele vai ser sempre criança. No caso dele 
a terapeuta ajudou muito os meus pais entenderem que a sexualidade não precisa ser vivida numa relação. [...]. Aí trabalhou com ele e os meus pais a importância de deixar ele, de ensinar a viver isso desde que fosse em lugar com privacidade. Mas no meu caso, meio que ninguém acha que precisa fazer nada.

Kalel ressalta ainda que a vivência da sexualidade, assim como a educação sexual de autistas não oralizados é comprometida pela falta de investimento em formas de comunicação aumentativa e alternativa desde a infância:

Pais não sabem falar sobre sexo nem com os filhos não autistas. [...] Agora, se o autista é não verbal e não se investe em outras formas de comunicação, vão restringir as ferramentas pra orientação. No caso do meu irmão, a terapeuta usa pistas visuais pra explicar a rotina e as coisas do cotidiano e dá certo.

Luke (17 anos), assim como Kalel, possui um irmão com grave limitação de autonomia e comprometimento cognitivo e corrobora a importância de preparar os pais para essas questões:

o mais difícil foi meus pais, principalmente minha mãe entender isso. Ela ficava resmungando que nós somos crianças sem maldade. Aí eu ria. Sabe de nada. [...] E vou dizer, meu irmão está com 11 anos, mas eu já disse que ela fosse pra terapia pra aprender a respeitar a puberdade dele. Porque a minha, que falo, já foi difícil.

A maior necessidade de suporte, incluindo de comunicação, não deve ser considerado um empecilho para a educação sexual de pessoas autistas, como ressalta Violet (16 anos):

eu não falava até os 7 anos. Usei pictogramas durante um tempo. Hoje eu ainda tenho dificuldades de falar. Você está vendo. E é horrível as pessoas acharem que você não tem nada a dizer por que não fala. [...] não se pode ignorar a sexualidade de autistas que não falam. Entender isso e trabalhar isso na escola, na terapia e em casa é importante [...] pra proteger mesmo a pessoa e respeitar também. É horrível você não ser respeitada como pessoa só por que não fala ou não consegue se comunicar.

Por outro lado, uma menor dificuldade de comunicação não reduz necessariamente as demandas sobre a sexualidade. Naruto (15 anos) não apresenta dificuldades de oralização. Todavia, Naruto relata dificuldades de compreensão das normas sociais, que culminaram na sua estigmatização por seus pares. Sobre isso, ele narrou o seguinte episódio:

A professora pareceu confusa com a minha pergunta. E eu só disse que tinha dificuldade em deixar que me toquem, mas que eu queria ser tocado.
Ela me olhou com espanto e disse que eu não devia pensar nessas coisas. Mas eu não entendi por que eu não podia perguntar isso na aula se era aula de aparelho reprodutor.

Após discutir esse episódio em sua terapia e dialogar com seus pais, Naruto relata ter compreendido que:

eu tenho que ter cuidado, porque existem pessoas que podem abusar da minha sinceridade. As pessoas não costumam lidar bem com esses assuntos. Eu entendi isso, mas isso não muda como me sinto [...]. Pra piorar, eu ainda virei o estranho, o esquisito e isso não ajuda em nada.

Ao contrário de Naruto, Sakura refere uma aparente facilidade de socialização, associada à sua habilidade de performar:

Algumas de nós performam. A gente aprende a imitar as pessoas pra conseguir se inserir nos espaços. A gente que nasce mulher aprende a fingir pra se adaptar. É por isso que é mais difícil dar o diagnóstico em meninas.

A habilidade de "performar normalidade" usando palavras dela - não facilitou, contudo, a vivência relacional de Sakura:

Eu nunca tive um namorado. Eu queria, queria mesmo até porque eu tenho desejo de verdade. Mas eu entendi na terapia que eu não posso sair dizendo isso, porque me coloca numa situação de risco. Mas acontece que performar normalidade não é ser normal. Eu tenho muita seletividade alimentar, por exemplo. Então eu não saio com as pessoas pra comer pra elas não notarem. Esse tipo de coisa dificulta.

Para além da multiplicidade de demandas e vivências, Noa (17 anos) traz à baila a diversidade de orientação sexuais e identidade de gênero:

Já ouvi: você não pode ser trans, você é autista e não tem autista trans. Tem sim. E quando eu falo que sou não binária é que o pessoal surta.

Para Noa, o afastamento das regras sociais hegemônicas torna as pessoas autistas mais propensas a se perceberem com características não normativas de gênero do que neurotípicos:

se você parar pra refletir, essa divisão binária não faz sentido. É tudo construção. A gente pensa objetivo, então essas questões não pesam tanto pra gente.

Thanus (17anos), define-se como assexual heterorromântico, por não sentir atração sexual, mas ser capaz de se apaixonar por outras pessoas. Para o jovem, existe uma resistência da sociedade em admitir diferentes formas de experiência relacional. Quando essa vivência se associa ao autismo, contudo, a desqualificação passa a ser justificada pela deficiência: 
as pessoas riem quando eu falo que sou assexual heterorromântico. Desqualificam mesmo. $E$ como coisa que criança fala e ninguém leva a sério. Pois é. É a mesma coisa quando a gente é autista.

\section{Discussão}

Nossos resultados corroboram que, embora pessoas autistas se desenvolvam física e sexualmente de acordo com os estágios típicos de desenvolvimento, existem singularidades que não devem ser ignoradas. As experiências e as expressões da sexualidade de pessoas autistas são tão diversas quanto o espectro ${ }^{14}$, demandando uma estrutura de apoio adaptável e que considere necessidades, desejos, dificuldades e compromentimentos ${ }^{2,14}$.

Os resultados desta pesquisa, contudo, demonstram que não apenas as singularidades são ignoradas, como a própria sexualidade das pessoas autistas é frequentemente alvo de silenciamento ${ }^{15}$. A literatura atribui essa atitude à baixa expectativa quanto à possibilidade de envolvimento romântico e ao receio de estimular um desejo sexual que não se desenvolveria espontaneamente ${ }^{16}$. Paradoxalmente, existe o reconhecimento de expressões da sexualidade em autistas com maiores necessidades de suporte, com foco em sua inadequação social ${ }^{17}$.

Nesse ponto, cabe destacar como os processos discursivos são organizados para a construção de um significado único para a sexualidade da pessoa autista. A metáfora do "anjo azul", criticada por nossos informantes, cria uma congruência narrativa entre um modelo patologizante da deficiência e a sociedade disciplinar normativa9. Desse modo, a infantilização do autista com maior necessidade de suporte legitima a patologização de suas sexualidades ${ }^{6,9}$. Na medida em que a limitação é atribuída exclusivamente à condição de deficiência, parte-se da presunção de incapacidade, culminando em uma limitação de investimentos $^{6}$. Em contrapartida, o desinvestimento fomenta a limitação, prendendo a pessoa em círculo vicioso ${ }^{3,6}$.

A desqualificação de expressões sexuais e de gênero não normativas também surgem nos resultados, nos relatos de Noa e Thanus. Suas histórias, contudo, não se constituem exceções, sendo crescentes as evidências de que pessoas autistas apresentam maior diversidade sexual e de gênero em relação a pessoas neurotípicas ${ }^{17,18}$. O silenciamento sobre essas questões, tanto na academia como em elementos midiáticos em um momen- to em que o autismo ganhou destaque em séries e programas de televisão, corrobora o papel dos discursos - e dos silenciamentos - na construção da imagética social do autismo\%.

Esses processos discursivos culminam em um paradoxo essencial: a uniformidade da negligência por meio de uma construção dicotômica. Conquanto reconheça que algumas pessoas autistas necessitam de maior suporte educacional e terapêutico que outras, a Neurodiversidade refuta o binário que as dicotomiza em alto ou baixo funcionamento ${ }^{3}$. Não se ignora que alguns comportamentos podem expor pessoas autistas a situações críticas, tornando-as tanto vítimas como agressores potenciais ${ }^{2,9}$. Tampouco, o paradigma da Neurodiversidade pressupõe ausência de suporte terapêutico. Todavia, parte-se do pressuposto de que as limitações derivam de um misto entre questões subjetivas e ambientais ${ }^{3}$. Deste modo que as terapias devem ser individualizadas, baseadas em estratégias educativas e visando que cada pessoa desenvolva as habilidades necessárias para conhecer, controlar e explorar o próprio corpo $^{6,8}$.

Por outro lado, mesmo autistas com déficits mais sutis podem apresentar dificuldades de socialização e necessitar de suporte, incluindo uma educação sexual adaptada ${ }^{2,3,19}$. A dicotomia entre baixo e alto funcionamento, contudo, frequentemente culmina na negligência das necessidades específicas das pessoas enquadradas neste grupo ${ }^{3}$.

Cabe ressaltar que, embora nossos informantes apresentassem diferentes níveis de necessidade de suporte, este artigo apresenta como limitação a ausência de autistas não oralizados, evidenciando-se uma brecha a ser preencida por estudos futuros.

\section{Considerações finais}

Discutir o enquadramento metafórico hegemônico do autismo nos possibilita repensar como essas construções simbólicas orientam as ações educativas e terapêuticas, em um sentido dicotomico de patologização ou negligência.

Sendo, contudo, diversas e singulares as expressões da sexulidade de pessoas autistas, estas demandam uma estrutura de apoio adaptável e que considere suas particularidades. Essas medidas, contudo, requerem uma mudança paradigmática, sob risco de mudarmos na forma e não na estrutura. 


\section{Colaboradores}

AVM Brilhante idealizou e coordenou a pesquisa, coletou os dados e participou da análise dos dados e da escrita do artigo. LMA Filgueira, SVMU Lopes, NBS Vilar, LRM Nóbrega, AJMV Pouchain e LCG Sucupira participaram da elaboração do projeto, da análise dos dados e da escrita do artigo.

\section{Referências}

1. Rios C, Ortega F, Zorzanelli R, Nascimento RL. Da invisibilidade à epidemia: a construção narrativa do autismo na mídia impressa brasileira. Interface [periódico na Internet], 2015 [cited 2019 May 22]; 19(53):325-336. Available from: http://www.scielo.br/scielo.php?script=sci_arttext\&pid=S1414-32832015000200325\&lng=en\&nrm=iso

2. Parchomiuk M. Sexuality of Persons with Autistic Spectrum Disorders (ASD). Sex Disabil [serial on the Internet], 2019 [cited 2019 May 10]; 37:259274. Avaiable from: https://link.springer.com/article/10.1007/s11195-018-9534-z?shared-article-renderer

3. Den Houting J. Neurodiversity: An insider's perspective, Autism [serial on the Internet], 2019 [cited 2020 Apr 10]; 23(2):271-273. Available from: https://journals.sagepub.com/doi/full/10.1177/1362361318820 762?url_ver $=$ Z39.88-2003\&rfr_id=ori:rid:crossref. org\&rfr_dat $=$ cr_pub\%3dpubmed

4. Beddows N, Brooks R. Inappropriate sexual behavior in adolescents with autism spectrum disorder: what education is recommended and why. Early Interv Psychiatry [serial on the Internet], 2016 [cited 2019 May 24]; 10(4):282-289. Available from: https://www.ncbi. nlm.nih.gov/pubmed/26265030

5. Kapp SK, Steward R, Crane L, Elliott D, Elphick C, Pellicano E, Russell G. 'People should be allowed to do what they like': Autistic adults' views and experiences of stimming. Autism serial on the Internet], 2019 [cited 2020 Apr 12]; 23(7):1782-1792. Available from: https://journals.sagepub.com/doi/full/10.11 77/1362361319829628?url_ver=Z39.88-2003\&rfr_ id=ori:rid:crossref.org\&rfr_dat=cr_pub\%3dpubmed

6. Silberman S. Neurotribes: The legacy of autism and the future of neurodiversity. New York: Penguim Random House; 2015.

7. Pantazakos T. Treatment for whom? Towards a phenomenological resolution of controversy within autism treatment. Stud Hist Philos Biol Biomed Sci [serial on the Internet]. 2019 [cited 2019 May 22]; 77:101176. Available from: https://www.sciencedirect.com/science/article/abs/pii/S1369848618301018?via\%3Dihub

8. Sala G, Pecora L, Hooley M, Stokes MA. As Diverse as the Spectrum Itself: Trends in Sexuality, Gender and Autism. Curr Dev Disord Rep [serial on the Internet], 2020 [cited 2020 Apr 13]; 10.1007/s40474-020-001901. Avaiable from: https://doi.org/10.1007/s40474-02000190-1

9. O'Reilly M, Lester JN. Social Constructionism, Autism Spectrum Disorder, and the Discursive Approaches. In: O’Reilly M, Lester J, Muskett T, editors. A Practical Guide to Social Interaction Research in Autism Spectrum Disorders. The Language of Mental Health. London: Palgrave Macmillan; 2017. p. 61-85.

10. Lopes SVMU, Vilar NBS, Pouchaim AJMV, Sucupira LCG, Nóbrega LRM, Brilhante AVM. Transtorno do Espectro Austista e Sexualidade. Atas CIAIQ 2018; 2:1175-1180. 
11. Endres RG. O Fenótipo Ampliado do Autismo em genitores de crianças com Transtorno do Espectro Autista - TEA [Internet]. Psic Teor e Pesq [periódico da Internet]. 2015 [acessado 2019 Maio 23]; 31(3):285-292. Disponível em: http:// www.scielo.br/scielo.php?script $=$ sci_arttext\&pid=S0102-37722015000300285\&lng=en\&nrm=iso

12. Minayo MCS. O Desafio do conhecimento: pesquisa qualitativa em saúde. São Paulo: Hucitec; 2014.

13. Bardin L. Análise de conteúdo. São Paulo: Edições 70; 2016

14. Sala G, Pecora L, Hooley M, Stokes MA. As Diverse as the Spectrum Itself: Trends in Sexuality, Gender and Autism. Curr Dev Disord Rep [serial on the Internet]. 2020 [cited 2020 Apr 13]. Avaiable from: https://doi. org/10.1007/s40474-020-00190-1

15. Holmes LG, Strassberg DS, Himle MB. Family Sexuality Communication: Parent Report for Autistic Young Adults Versus a Comparison Group. J Autism Dev Disord [serial on the Internet]. 2020 [cited $2020 \mathrm{Apr}$ 13]. Avaiable from: https://www.ncbi.nlm.nih.gov/ pubmed/32056120

16. Holmes LG, Himle MB, Strassberg DS. Parental romantic expectations and parent-child sexuality communication in autism spectrum disorders. Autism [serial on the Internet]. 2016 [cited 2019 May 23]; 20(6):687-699. Avaiable from: https://www.ncbi.nlm. nih.gov/pubmed/26408632

17. Glidden D, Bouman WP, Jones BA, Arcelus J. Gender Dysphoria and Autism Spectrum Disorder: A Systematic Review of the Literature. Sex Med Rev [serial on the Internet]; 2016 [cited 2019 May 23]; 4(1):3-14. Avaiable from: https://www.sciencedirect.com/science/article/abs/pii/S2050052115000049

18. Strang JF, Janssen A, Tishelman A, Leibowitz SF, Kenworthy L, McGuire JK, Edwards-Leeper L, Mazefsky CA, Rofey D, Bascom J, Caplan R, Gomez-Lobo V, Berg D, Zaks Z, Wallace GL, Wimms H, Pine-Twaddell E, Shumer D, Register-Brown K, Sadikova E, Anthony LG. Revisiting the Link: Evidence of the Rates of Autism in Studies of Gender Diverse Individuals. J Am Acad Child Adolesc Psychiatry [serial on the Internet], 2018 [cited 2019 May 23]; 57(11):885887. Avaiable from: https://www.ncbi.nlm.nih.gov/ pubmed/30392631

19. Barnett JP, Maticka-Tyndale E. Qualitative Exploration of Sexual Experiences Among Adults on the Autism Spectrum: Implications for Sex Education. Perspect Sex Reprod Health [serial on the Internet], 2015 [cited 2019 May 23]; 47(4):171-179. Available from: https://www.ncbi.nlm.nih.gov/pubmed/26418175

Artigo apresentado em 04/05/2020

Aprovado em 15/06/2020

Versão final apresentada em 17/06/2020

Editores chefes: Maria Cecília de Souza Minayo, Romeu Gomes, Antônio Augusto Moura da Silva 
